GALACTIC BACKGROUND STARLIGHT FROM UV TO IR: OBSERVATIONS AND MODELS 


\title{
OPTICAL OBSERVATIONS OF GALACTIC AND EXTRAGALACTIC LIGHT: IMPLICATIONS FOR GALACTIC STRUCTURE
}

\author{
Gary N. Toller \\ General Sciences Corporation \\ 6100 Chevy Chase Drive \\ Laurel, MD 20707 USA
}

\begin{abstract}
A historical review of integrated starlight, diffuse galactic light, and extragalactic light studies is presented. Together, these components compose the "background light." Methods ranging from star counts to space-based photometric surveys have succeeded in quantifying the contribution of each component of the background. Integrated starlight is the dominant component. The contribution of diffuse galactic light in the general interstellar medium peaks slightly off the galactic plane and declines toward higher latitudes. The extragalactic light has been determined from both galaxy counts and photometric methods. The blue and red intensity and B-R color distribution of background light have been mapped. The relation between galactic structure and background light measurements is established. The distribution of interstellar extinction is the primary regulator of the brightness. However, spiral arm and stellar distribution effects are discerned in Carina and Sagittarius. The sun lies $13 \mathrm{pc}$ north of the galactic plane as defined by brightness and dust distributions.
\end{abstract}

\section{INTRODUCTION}

Separated by millenia, ancient and modern peoples have sought to comprehend the heavens. Our knowledge of the galaxy becomes more firmly established as human curiosity, coupled with technology, probe the Milky Way. Only recently have astronomers quantitatively separated the light of the night sky into its components.

Background light consists of integrated starlight $\left(m_{v}>6.5\right)$, diffuse galactic light (starlight scattered by dust), and extragalactic background light. The typical brightness of various sources contributing to the night sky brightness is provided in Table 1 . The historical $S_{10}$ unit is a measure of brightness. Brightness expressed in $S_{10}(V)_{G 2 V}$ equals the equivalent number of tenth magnitude $(V)$ solar-type stars per square degree. In physical units, $1 S_{10}(V)_{G 2 V}=$ $1.16 \times 10^{-9} \mathrm{erg} \mathrm{cm}^{-2} \mathrm{~s}^{-1} \mathrm{sr}^{-1} \AA^{-1}$ at blue wavelengths, $1.09 \times 10^{-9} \mathrm{erg} \mathrm{cm}^{-2} \mathrm{~s}^{-1} \mathrm{sr}^{-1} \AA^{-1}$ in the red. Other forms of the $S_{10}$ unit are described by Toller, Tanabe, and Weinberg (1987).

TABLE 1. Typical Brightness of Selected Astronomical Sources as Viewed from Earth

\begin{tabular}{lc}
\hline \hline & Typical Brightness \\
Component & $S_{10}(V)_{G 2 V}$ \\
\hline Full Moon & $9 \times 10^{10}$ \\
Airglow Continuum & 50 \\
Zodiacal Light (on ecliptic) & 200 \\
Zodiacal Light (off ecliptic) & 90 \\
Bright Stars ( $\left.m_{v}<6\right)$ & 20 \\
Integrated Starlight (galactic plane) & 200 \\
Integrated Starlight (off galactic plane) & 50 \\
Diffuse Galactic Light & 20 \\
Cosmic Light & 1 \\
\end{tabular}

21

S. Bowyer and C. Leinert (eds.), The Galactic and Extragalactic Background Radiation, 21-34.

(c) 1990 IAU. Printed in the Netherlands. 


\section{HISTORICAL STAR COUNTS AND PHOTOMETRIC SURVEYS}

Most of the background light comes from numerous faint stars. Galileo observed the Milky Way band in 1609 and discerned it to be composed of myriad individual stars. William Herschel counted stars to approximately $m_{v}=14$ two hundred years ago and quantitatively demonstrated the disk shape of our galaxy. A systematic survey of 206 Selected Areas (hereafter SAs) was carried out by Kapteyn starting in 1906. Unaware of interstellar extinction, Kapteyn's star count research indicated that the sun lay in the disk of the Milky Way near the center of a small, flattened galaxy. Star count analysis by Bok (1931) indicated that the local system (Bok, 1937) exhibits enhanced stellar density.

Bottlinger (1932) integrated Mount Wilson star count data in the SAs to obtain integrated starlight as a function of galactic latitude. The raw Mount Wilson counts are reliable for $b^{\text {II }}>20^{\circ}$. At lower latitudes the discrepancies between the Mount Wilson and Gröningen star counts are well documented (Henyey and Greenstein, 1941; Roach, 1964; Lillie and Witt, 1976). The application of star counts to the determination of the stellar content in the galaxy has proved highly successful (Blaauw and Schmidt, 1965).

In 1933, Pannekoek measured the brightness of the Northern Milky Way based on photometry of photographs taken by Max Wolf at Heidelberg. This work was extended to the Southern Milky Way by Pannekoek and Koelbloed (1949). Taken together, these works presented isophotes at all longitudes for latitudes $\left|b^{\mathrm{I}}\right| 15^{\circ}$. This work is historically important, but calibration problems caused the brightness levels to be low (Peters, 1970). Additionally, the derived intensities include the zodiacal light, airglow emission, and atmospheric scattered light.

Elvey and Roach (1937, hereafter ER) used a photoelectric photometer to map the sky at $4500 \AA$ with $5^{\circ}$ resolution. They presented figures of the excess brightness with respect to Bottlinger's mean star count data. The total background light can be obtained by adding Bottlinger's stellar component to Figure 13 and Table 3 of ER. The ER absolute calibration was suspected to be high by Roach and Smith (1964) and Wolstencroft and Rose (1966). Comparison with modern space-based photometry also leads one to reject use of the ER study as a determinant of the background light or of any of its components.

\section{MODERN STAR COUNTS AND PHOTOMETRIC SURVEYS OF BACKGROUND LIGHT}

The Pioneer 10 and 11 space probes, launched in 1972 and 1973, respectively, each carried an imaging photopolarimeter. After passing through the asteroid belt, the background light was mapped at blue and red wavelengths in the absence of foreground contamination from the Earth or zodiacal light. The experiment has been described elsewhere (Weinberg et al., 1984; Hanner et al., 1974; Toller, 1981).

Isophotes derived from this study can be found in Weinberg (1981) and Toller, Tanabe, and Weinberg (1987). Background light values have been tabulated by Toller (1981). Using the Pioneer 10 sky survey at blue wavelengths as a standard, we compare Pioneer data to other studies, starting with Elsässer and Haug (1960, hereafter EH). EH presented isophotes of the background light for the region $b^{\mathrm{I}}<30^{\circ}$ using photoelectric surface photometry measurements from South Africa and Switzerland. The absolute scale of the photometry has been questioned by Megill and Roach (1961), Roach and Smith (1964), and Sharov and Lipaeva (1973). It is generally believed that the EH brightness at blue wavelengths is too low. 
If we make the limiting magnitude compatible with Pioneer $\left(m_{v} \geq 6.5\right)$ by using the B-V distribution over the sky from EH in conjunction with the stellar contribution as a function of visual magnitude (Roach and Megill, 1961), and compare these two studies in $S_{10}$ units, a least squares fit relation is obtained:

$$
\mathrm{EH}=0.65 \times \text { Pioneer }+42.4 \text {. }
$$

The constants in this equation reflect the two types of errors inherent in night sky photometry. Because the background light is determined by subtracting atmospheric line and continuum emission, atmospheric extinction and scattering, and zodiacal light, errors in the estimation of any component propagate to the background light. This is the subtractive error. Mistakes in absolute calibration cause multiplication errors in the intensity levels and indirectly cause subtraction errors.

Roach and Megill (see also Megill and Roach, 1961) integrated the GR43 star counts at Kapteyn's 206 SAs (van Rhijn, 1925) to obtain the integrated starlight. Star counts by Seares et al. (1925) in SAs 1-139 using the Mount Wilson star catalogs deviate substantially from those of GR43 as the galactic plane is approached. Integrated starlight levels derived from the former are systematically lower than those derived from the latter. The nonuniformity of stellar distribution and of extinction at low latitudes are major sources of error in star count studies. In addition, the van Rhijn study had to contend with slight inaccuracies in the magnitude scale in the southern SAs.

Using observations at $5300 \AA$, Roach and Smith suggested that the Roach and Megill data be raised by $26 \%$. Sternberg and Ingham (1972) found that Roach and Megill's data should be increased by $10 \%$. However, even without raising the values of Roach and Megill, their brightness values exceed those of EH. Sharov and Lipaeva applied corrections to the catalogs used by van Rhijn. Their new counts in the SAs culminated in brightnesses $33 \%$ below those of Roach and Megill on the average. These discrepancies highlight some of the difficulties associated with star count and photometric studies. The relatively sparse spatial coverage of the star counts and major differences, for example, between GR43 and Mt. Wilson star count studies of similar areas make these data unreliable at low galactic latitudes.

The generally higher values of Pioneer are attributable to the presence of diffuse galactic light and extragalactic light in photometric observations. Integrated starlight is the brightest component of background light and should not differ from it by more than $10 \mathrm{~S}_{10}(V)_{G 2 V}$ at high $\left(|\mathrm{b}|>60^{\circ}\right)$ galactic latitudes. At high latitudes, Pioneer photometry is consistent with both studies. At $\left|b^{I I}\right|<30^{\circ}$, Pioneer values are more consistent with Sharov and Lipaeva.

Lillie (1968) mapped approximately $1 / 4$ of the sky using a rocket-borne photoelectric photometer. His data have a variable spatial resolution of $2-60^{\circ}$. Converting the $4100 \AA$ data to common units and limiting magnitude $m_{v}=6.5$, one obtains a least squares fit relation:

$$
\text { Lillie }=1.29 \times \text { Pioneer }+0.8 \text {. }
$$

Classen published isophotes of background light at $4300 \AA$ derived from Pfleiderer's photoelectric measurements. This ground-based study provides nearly full sky coverage at high spatial resolution. Intensity levels from Pioneer should be slightly above Classen's values, due to a slight difference in limiting magnitudes at intermediate and high latitudes. A comparison reveals that Pioneer brightness values exceed Classen's values by $8 \%$. 


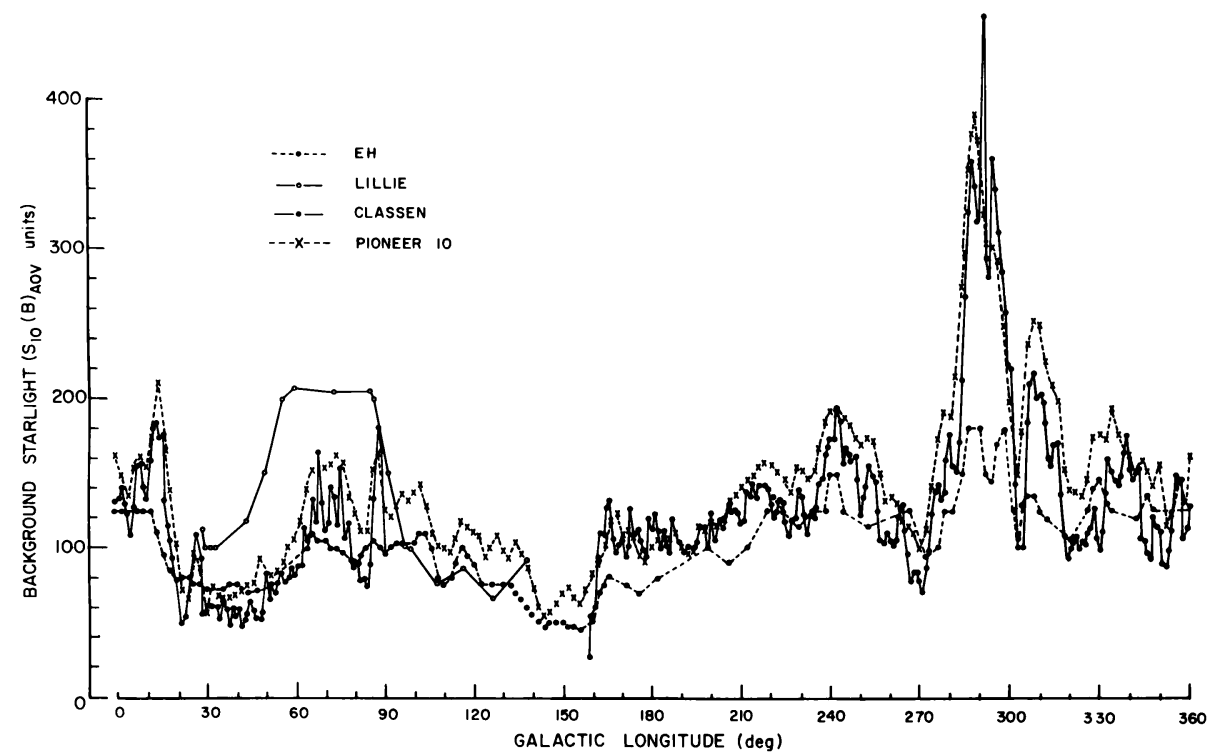

Figure 1. Photometric survey of background light at blue wavelengths. $b^{\text {II }}=0$.

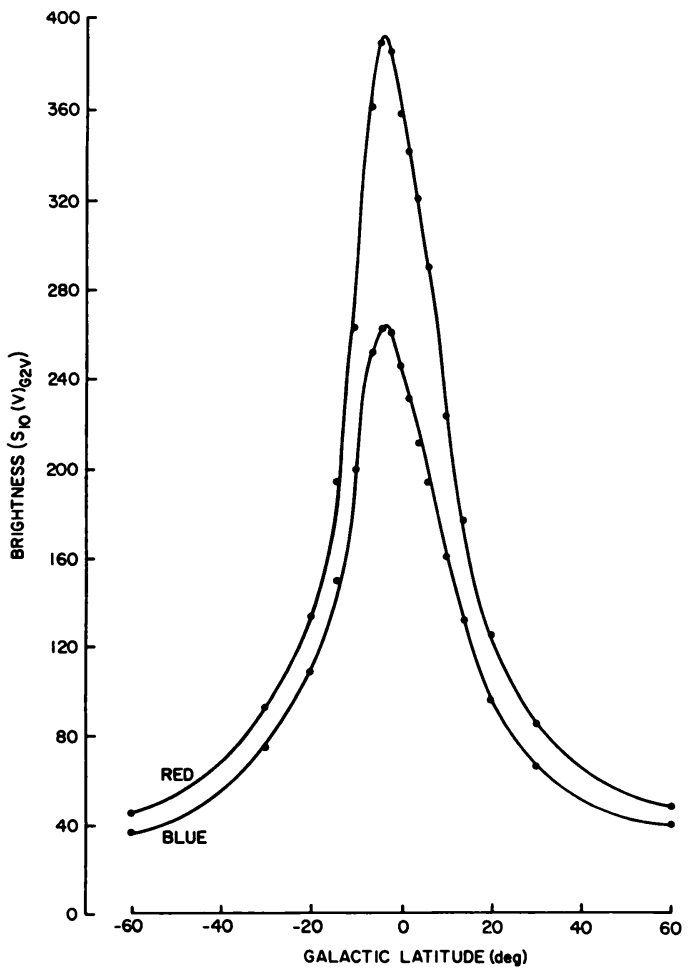

Photometry from the Helios 1 spacecraft was used by Hanner, Leinert, and Pitz (1978) to derive Milky Way intensities in Scorpius with a $15 \%$ uncertainty. In arriving at these values, Classen's zodiacal light brightness values were used. Pioneer data exceeds Helios background light levels by $16 \%$.

A scan of the galactic plane, including data from $\mathrm{EH}$, Lillie, Classen, and Pioneer, is depicted in Figure 1. The general trend of background light as a function of galactic latitude appears in Figure 2. Further details of these studies are provided by Toller (1981).

Figure 2. Pioneer 10 observations of background light as a function of galactic latitude. 


\section{DETERMINATION OF INTEGRATED STARLIGHT AND DIFFUSE GALACTIC LIGHT}

Integrated starlight is the dominant component of background starlight, even at low galactic latitudes where the contribution of diffuse galactic light (hereafter, DGL) becomes significant. Comparisons between background light studies and star counts provide a means for the separation of integrated starlight and DGL, thereby allowing the determination of fundamental information on each. The values obtained for the integrated starlight and DGL provide important limits which constrain theoretical galactic structure models.

A chronology of observational DGL studies undertaken at blue wavelengths appears in Table 2. The major findings are also summarized. Studies confined to one small area (e.g., a dark cloud) are excluded from the scope of this review.

Accurate DGL information at blue wavelengths can be obtained from comparison of Pioneer photometry with the detailed star counts of Roach and Megill (RM), Sharov and Lipaeva (SL), or Tanabe and Mori $(1971,1976)$. The SL study (limiting magnitude $m_{B}=8$ ) produces brightness levels $14.5 \%$ below RM if the limiting magnitudes are made compatible by applying RM integrated starlight as a function of magnitude. Because the work of SL supersedes RM and their data are presented precisely at the 206 SAs, it is preferable to utilize the SL values, corrected to $m_{v} \geq 6.5$, at low latitudes. In this paper, the DGL values derived from $\mathrm{RM}$ and SL at SAs above $\left|b^{\mathrm{II}}\right|=30^{\circ}$ are obtained by averaging their starlight values.

Tanabe and Mori (1971) have developed and used a device to determine the number of stars and the associated size-magnitude relationships for images on blue and red prints of the Palomar Sky Survey plates. Integration over these data gives the integrated starlight plus the contribution of discrete galaxies. Tanabe (1973) and Tanabe and Mori $(1976,1979)$ compared integrated starlight to Pioneer 10 observations for several directions.

Subtracting starlight from Pioneer 10 photometric measurements leaves a residual that equals DGL plus extragalactic background light (hereafter, EBL). The relation between DGL+EBL and galactic latitude $b^{\text {II }}$ is depicted in Figure 3. The midpoints and standard deviations were obtained by grouping the RM, SL, and Tanabe data into 25 latitude bins and calcu-

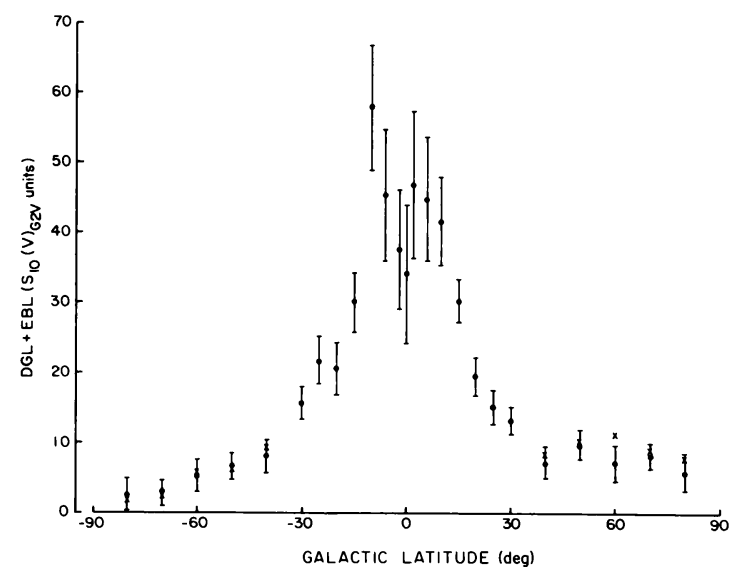

Figure 3. Latitude dependence of DGL+EBL. lating an average, weighted by the error of each measurement. A peak at $5^{\circ}<|b|<15^{\circ}$ and a drop in the contribution of DGL at high latitudes are the salient features. Variations from the often used assumption of a constant, latitudeindependent ratio are evident. The results presented here agree with the results of an investigation by Dumont et al. (1970). At $5020 \AA$, they examined the DGL at latitudes $30^{\circ}<\left|b^{I I}\right|<60^{\circ}$, concluding that as $\left|b^{\mathrm{II}}\right|$ increased, the DGL decreased by $\leq .3 \mathrm{~S}_{10}(V)_{G 2 V}$ per degree of latitude and that the ratio of DGL to starlight does not exceed .25 at these latitudes. 
TABLE 2. A Chronology of Observational Diffuse Galactic Light Studies

\begin{tabular}{|c|c|c|}
\hline Author/Year & Region Observed & Results \\
\hline Elvey and Roach, 1937 & $\left|b^{I}\right|<40^{\circ}$ & $\begin{array}{l}\text { DGL strongest in Sagittarius, } \\
\text { faintest in Auriga }\end{array}$ \\
\hline $\begin{array}{l}\text { Henyey and Greenstein, } \\
1940,1941\end{array}$ & $\begin{array}{l}l^{1}=40^{\circ}, 140^{\circ} \\
\left|b^{1}\right|<30^{\circ}\end{array}$ & S. Milky Way brighter \\
\hline Kreiken, 1941 & Kapteyn SAs & DGL correlates with integrated starlight \\
\hline van Houten, 1961 & $b=0^{\circ}$ & $\mathrm{DGL}=12 \mathrm{~S}_{10}(p g)_{A O V}$ \\
\hline Schmidt and Leinert, 1966 & $l^{I I}=101^{\circ}$ & DGL polarization detected \\
\hline $\begin{array}{l}\text { Wolstencroft and Rose } \\
1966\end{array}$ & $\begin{array}{l}l^{I}=53,66,164^{\circ} \\
\left|b^{I}\right|<15^{\circ}\end{array}$ & $<\mathrm{DGL}>=72 \mathrm{~S}_{10}(B)_{A O V}$ \\
\hline Roach, 1967 & SAs & $\begin{array}{l}\text { Ratio of DGL to direct starlight } \\
\text { is latitude dependent }\end{array}$ \\
\hline van Houten, 1967 & See Reference & Existence of DGL uncertain \\
\hline Witt, 1968 & $\begin{array}{l}\text { Cygnus, Taurus- } \\
\text { Auriga }\end{array}$ & $\begin{array}{l}\text { B-V (Cygnus DGL) }=.57, .44 \text { at } \\
b^{I I}=0^{\circ}, 25^{\circ} \text { respectively }\end{array}$ \\
\hline Lillie, 1968 & $\begin{array}{l}\text { Regions Between } \\
l^{11}=29,137^{\circ}\end{array}$ & $\begin{array}{l}87 S_{10}(B)_{A O V} \text { peak intensity at } \\
b^{\mathbb{I}}=0^{\circ} .(B-V)_{D G L}=.51\end{array}$ \\
\hline Weinberg, 1969 & $b^{1}=0^{\circ}, l^{1}=23^{\circ}$ & DGL polarization detected \\
\hline Peters, 1970 & $\left|b^{\mathbf{I}}\right|<12^{\circ}$ & Ratio DGL to direct starlight is $20-30 \%$ \\
\hline Dumont et al., 1970 & $30^{\circ}<\left|b^{\text {II }}\right|<60^{\circ}$ & $\begin{array}{l}\text { Slope from } 30^{\circ} \text { to } 60^{\circ}=-0.3 \mathrm{~S}_{10}(V) \\
\text { per degree. DGL }=25 \% \text { direct starlight }\end{array}$ \\
\hline Mattila, 1970 & $\begin{array}{l}\text { Dark Nebulae in } \\
\text { S. Coalsack, Libra }\end{array}$ & $\begin{array}{l}\text { Coalsack: DGL }=10-50 S_{10}(B)_{A O V} \text {; } \\
\text { Libra: } D G L=14 S_{10}(B)_{A O V}\end{array}$ \\
\hline Kondraijeva, 1971 & $\begin{array}{l}l^{I I}=34^{\circ} .4 \\
b^{I I}=.5^{\circ}\end{array}$ & $\mathrm{DGL}=10.2 \mathrm{~S}_{10}(p g)_{A O V}$ \\
\hline Sparrow and Ney, 1972 & $l^{\mathrm{II}}=35,210^{\circ}$ & DGL polarization detected \\
\hline Roach et al., 1972 & $\begin{array}{l}\mathrm{RA}=257-336^{\circ} \\
\mathrm{DEC}=20^{\circ} 42^{\prime}\end{array}$ & $\begin{array}{l}\text { Asymmetry exists with respect to the galactic } \\
\text { plane. DGL emanates from discrete sources }\end{array}$ \\
\hline Witt and Lillie, 1973 & 29 SAs & DGL correlates with integrated starlight \\
\hline Lillie and Witt, 1976 & $65^{\circ}<l^{I 1}<145^{\circ}$ & $\begin{array}{l}\mathrm{DGL}=47 \mathrm{~S}_{10}(4250 \AA) \text { at }|b|=1^{\circ}, \\
\text { Ratio of DGL to starlight }=42 \%\end{array}$ \\
\hline Eroshevich, 1978 & $l=67 \%, b=-3^{\circ}$ & $20 \pm 14 S_{10}(4500 \AA)_{A O V}$ \\
\hline Toller, 1981 & 193 SAs & $\begin{array}{l}\text { Latitude dependence of DGL and ratio to } \\
\text { starlight found }\end{array}$ \\
\hline
\end{tabular}

The relatively small DGL levels derived lend support to the preference for Pioneer 10 background starlight measurements over those obtained by Classen or from Helios. The larger brightness levels measured from Pioneer are needed to produce the moderate DGL residuals of Figure 3. Comparison between the observed DGL levels at $|b|>10^{\circ}$ and the neutral hydrogen column density (Heiles, 1975) shows a correlation which suggests that the DGL varies according to the extinction along the line of sight. The relationship at $4407 \AA$ is (Toller, 1981)

$$
D G L\left(S_{10}(V)_{G 2 V}\right)=2.4 \times 10^{-20} N_{H I} \text { atoms } \mathrm{cm}^{-2} \text {. }
$$




\section{THE EXTRAGALACTIC BACKGROUND AT OPTICAL WAVELENGTHS}

The extragalactic background light (EBL) is the faintest celestial background component when nearby, discrete galaxies are excluded from consideration. Quantitative determination of the EBL is a formidable challenge in view of its negligible contribution to the light of the night sky.

Investigations of the EBL have been based on either galaxy counts or photometric observations. A summary of representative results is provided in Table 3. Conversion to a uniform $S_{10}$ unit in the last column facilitates the comparison between disparate data sets. Photometric surveys provide an upper limit to the EBL based on the errors inherent in the subtractive method. Galaxy counts and differential photometry (Boughn and Kuhn, 1986; Mattila, 1989, this volume) provide a measurement of the EBL. Details of the various methodologies and of the studies listed in Table 3 can be found in Toller (1983) and Mattila (1989, this volume).

TABLE 3. Summary of EBL Studies

\begin{tabular}{lllr}
\hline \multicolumn{1}{c}{ Source } & \multicolumn{1}{c}{ Method } & \multicolumn{1}{c}{ Results } & $\mathrm{S}_{10}(V)_{G 2 V, \lambda}$ \\
\hline de Vaucouleurs, 1949 & galaxy count & $50 \mathrm{~S}_{15}(\lambda=p g)$ & 0.9 \\
Shane and Wirtanen, 1967 & galaxy count & $2 \times 10^{-10} \mathrm{Suns} \mathrm{pc}^{-3}$ & 0.7 \\
Roach and Smith, 1968 & photometry & $<5 \mathrm{~S}_{10}(5300 \AA)$ & $<5.2$ \\
Lillie, 1968 & photometry & $<2 \mathrm{~S}_{10}(\lambda=B)$ & $<4.5$ \\
Tanabe and Mori, 1971 & galaxy count (NGP) & $0.1 \mathrm{~S}_{10}(\lambda=p g)$ & 0.2 \\
Tanabe and Mori, 1971 & galaxy count (NEP) & $0.5 \mathrm{~S}_{10}(\lambda=p g)$ & 1.0 \\
Tanabe and Mori, 1976 & galaxy count (NGP) & $0.6 \mathrm{~S}_{10}(\lambda=p g)$ & 1.1 \\
Tanabe and Mori, 1976 & galaxy count (SGP) & $0.6 \mathrm{~S}_{10}(\lambda=p g)$ & 1.1 \\
Mattila, 1976 & photometry & $10 \mathrm{~S}_{10}(4000 \AA)$ & 25 \\
Dube et al., 1977 & photometry & $<3.4 \mathrm{~S}_{10}(5115 \AA)$ & $<4$ \\
Spinrad and Stone, 1978 & photometry & $<5 \mathrm{~S}_{10}(\lambda=B)$ & $<11.3$ \\
Peterson et al., 1979 & galaxy count & $0.17 \mathrm{~S}_{10}(4400 \AA)$ & 0.33 \\
Kirschner et al., 1979 & galaxy count & $10^{8} \mathrm{Suns} \mathrm{Mpc}^{-3}$ & 0.35 \\
Tyson and Jarvis, 1979 & galaxy count & $0.39 \mathrm{~S}_{10}(\lambda=J)$ & 0.7 \\
Tanabe and Mori, 1979 & galaxy count & $0.8 \mathrm{~S}_{10}(\lambda=p g)$ & 1.6 \\
Toller, 1981 & photometry & $1.3 \mathrm{~S}_{10}(V)_{G 2 V \text { at4407 }} \AA$ & $1.3 \pm 1.3$ \\
\hline
\end{tabular}

The subtractive method has been applied to Pioneer 10 background light measurements at blue wavelengths to extract the EBL. Observations from the outer solar system eliminate atmospheric and interplanetary dust effects. The most accurate EBL determinations derive from observations near the galactic poles, where integrated starlight and DGL are minimized. This calculation of DGL+EBL at the galactic poles employs star count data from $\left|b^{\mathrm{II}}\right|>70^{\circ}$ (Roach and Megill; Sharov and Lipaeva; Tanabe (1973, this volume), and unpublished work). The average value of EBL+DGL is $3.3 \pm 1.2 \mathrm{~S}_{10}(V)_{G 2 V}$ at $4407 \AA$. The DGL at the galactic poles is $2.0 \pm .4$, determined by Toller (1981). The standard deviation is calculated from the errors in each component employed in the subtractive method. The EBL at blue wavelengths equals $1.3 \pm 1.3 \mathrm{~S}_{10}(V)_{G 2 V}$. The integrated light from discrete galaxies adequately explains this intensity. Further details can be found in Toller $(1981,1983)$ and Tyson $(1988 ; 1989$, this volume). 


\section{THE CORRELATION BETWEEN BACKGROUND LIGHT AND GALACTIC STRUCTURE}

The application of visual wavelength photometry for the task of unraveling the Milky Way's spiral structure has sparked controversy since its inception. The crux of the problem lies in the data interpretation. Should one expect a brightness enhancement when viewing a spiral arm tangentially, due to an increase in star number density, or a brightness minimum due to increased extinction?

Different investigators who attempted to relate surface brightness levels to spiral structure features reached opposing conclusions. These historic studies include Kreiken (1936), Elsässer and Haug (1960), Isserstedt and Schmidt-Kaler (1964), Sharov (1964), Behr (1965), Pavlovskaya and Sharov (1971), Elsässer, Neckel, and Scheffler (1965), Neckel (1968), Uranova (1969), Peters (1970), Sharov (1971), and Zavarzin (1978). Contradictions among previous studies, plus recent advances in our knowledge of background light, the extinction distribution, and Milky Way spiral structure, indicate that the relationship between these features should be reexamined. To this end we analyze the blue and red photometry from Pioneer 10, presented in Figure 4. The curves were drawn by plotting the maximum brightness occurring at each longitude, a procedure employed by Elsässer and Haug. Note the excellent correspondence between the surface brightness curves at blue and red wavelengths. Major peaks in Figure 4 centered near $l=2,26,69,100,244,288,310$, and $330^{\circ}$ are indicated by arrows. Prominent minima occur at $30,150,302$, and $338^{\circ}$.

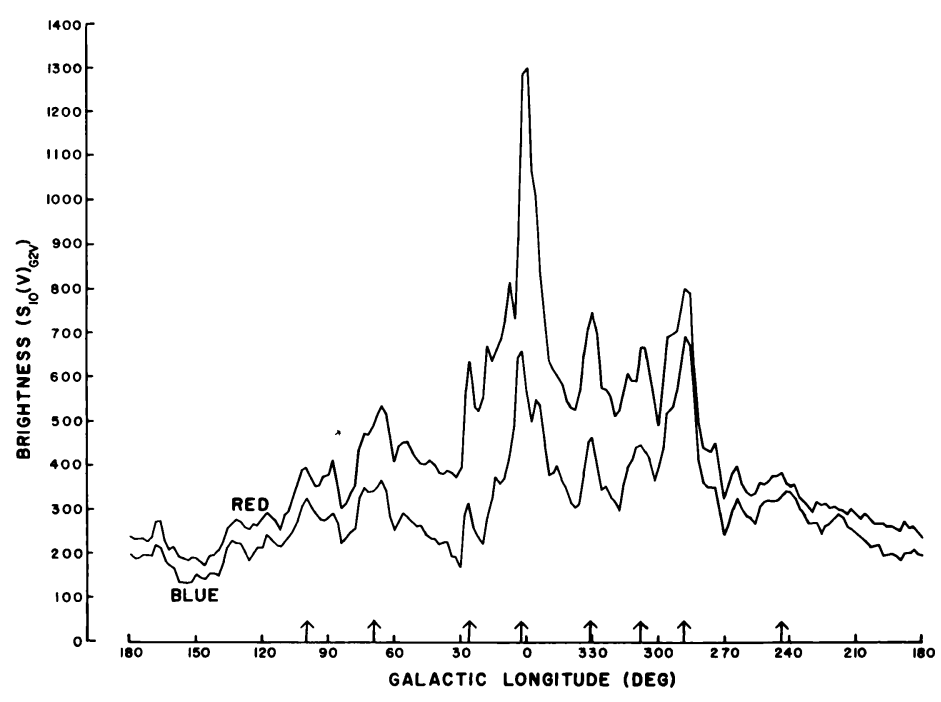

Figure 4. Pioneer 10 two-color observations of background light maxima as a function of galactic longitude.

The distribution of Pioneer $10 \mathrm{~B}-\mathrm{R}$ color index values near the galactic plane is plotted in Figure 5. Several salient features are worth noting. B-R exceeds the solar color (1.17) everywhere and increases as the galactic center is approached. There is a rough symmetry with respect to $l=12^{\circ}, b=1^{\circ}$. The smallest values are found in the range $l=210^{\circ}-240^{\circ}$, a position that correlates well with low extinction (FitzGerald, 1968). 


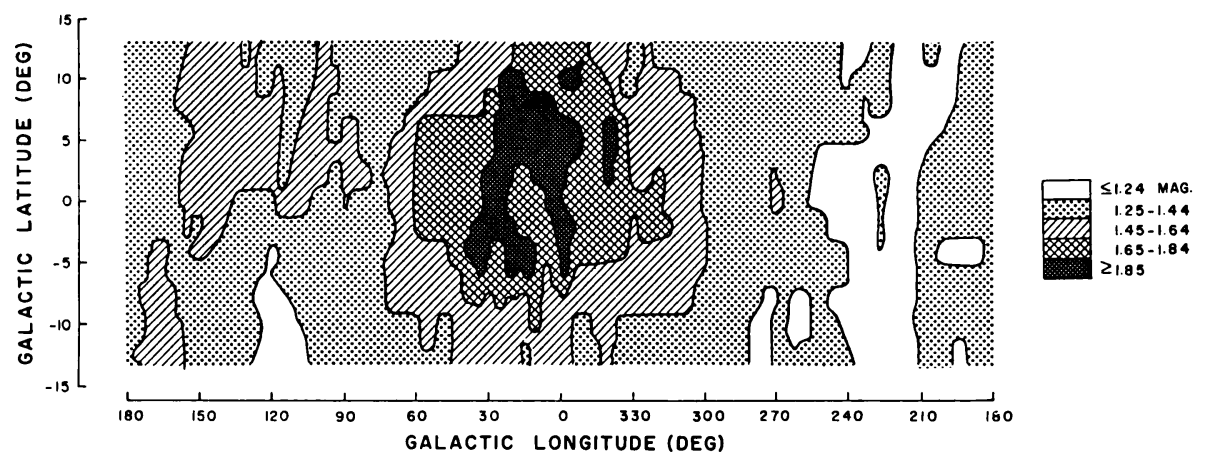

Figure 5. Pioneer 10 observations of color index B-R near the galactic plane.

To test for a relationship between the features of Figure 4 and the presence of interstellar extinction, published color excess and HI survey data were compiled (Toller, 1981). The broad peak in the range $l=340 \ldots 0 \ldots 20^{\circ}$ results from the influence of increased star density at these longitudes, especially within the constellation Sagittarius. Low extinction in the direction $l=2^{\circ}, b=-4^{\circ}$ for distances less than $1 \mathrm{kpc}$ strengthens the magnitude of the peak. The galaxy model of Toller (1981) predicts that the Sagittarius arm will also enhance the brightness at this longitude. The feature at $l=26^{\circ}$ appears only because the Scutum dark cloud complex at $l=20^{\circ}$ and $30^{\circ}$ causes surface brightness minima on either side of the $l=26^{\circ}$ "peak." A complete absence of low latitude transparency windows in the range $l=7-55^{\circ}, 121-175^{\circ}$ causes surface brightness levels at these longitudes to lie below the values found on the other side of the galactic center. The peak at $l=64-74^{\circ}$ corresponds to the line of sight along the inside of the Orion (local) arm. Light from young stars as well as from population II objects contributes strongly to lines of sight in this range which have low extinction relative to their neighbors. The extinction catalog confirms that a transparent region exists between $l=64^{\circ}$ and $74^{\circ}$ for $b$ in the range $2-4^{\circ}$. All directions between $l=64^{\circ}$ and $270^{\circ}$ traverse the Orion spiral arm, yet this longitude range is relatively dim and featureless in Figure 4, indica ting the dominant role of interstellar extinction in regulating the distribution of surface brightness. Peaks at $l=93^{\circ}, b=-2^{\circ}$ and $l=99^{\circ}, b=-6^{\circ}$ are caused by transparency channels. A dip at $l=150^{\circ}$ results from high extinction (Neckel, 1967; fields 73 and 200). Coincidentally, this direction corresponds to the line of sight passing perpendicular to the Orion arm. Numerous regions of low extinction exist in the range $l=223-250^{\circ}$, with the area between $240^{\circ}$ and $250^{\circ}$ having the largest extent in latitude. Clearly, the peak at $l=244^{\circ}$ is produced in a region characterized by unusually low extinction. The enhancement at $l=284-292^{\circ}$ corresponds with the direction of the Carina spiral feature. Low values of the extinction occur near $l=288^{\circ}, b=0-2^{\circ}$. The strength of this peak suggests a combination of low extinction and increased star number density in this direction. The Coalsack, present at $l=302^{\circ}$, correlates with the brightness minimum at this location. No anomalous extinction occurs near the $l=308^{\circ}$ peak, although relative to neighboring longitudes the extinction is lower at the peak. A slight extinction deficiency occurs near $l=332^{\circ}$ and the neighboring extinction is high. However, the magnitude of this feature indicates a local star density enhancement, as suggested by Peters, also contributes at this longitude. The "dip" at $l=338^{\circ}$ appears as such solely due to the presence of peaks on either side. The extinction in this region is normal. 
Evidence for some influence of spiral structure in the directions $l=64-74^{\circ}, 284-292^{\circ}$ is suggested by Figure 5. The rapid decrease of B-R values as we move from $60^{\circ}$ longitude to $80^{\circ}$ and the low values of B-R near $l=288^{\circ}$ can be explained by an increase of population I objects.

The dominance of interstellar extinction in determining background light levels is now firmly established. The irregular spatial distribution of interstellar dust (Krautter, 1980) causes peaks and dips in the brightness as we scan in longitude near the galactic plane. We have presented evidence here for the supplementary influence of spiral structure on background light measurements in the directions $l=2^{\circ}, 69^{\circ}, 288^{\circ}$. However, studying large scale spiral structure and the displacement of spiral arms from the mean plane through analysis of optical surface brightness measurements is unproductive.

\section{THE SUN'S DISTANCE FROM THE GALACTIC PLANE}

The determination of the position of the galactic plane has been pursued historically under the presumption that a particular class of objects defines the mean plane. Van Tulder's (1942) study of five classes of stellar objects with strong concentrations to the galactic plane indicated the sun lies $13.5 \pm 1.9 \mathrm{pc}$ north of the symmetry plane. Gum, Kerr, and Westerhout (1960) placed the sun $42 \mathrm{pc}$ above the mean plane of interstellar hydrogen. A similar undertaking by Davies (1960) culminated in a solar height $Z_{0}=22$ pc. Blaauw (1960) examined Cepheids with $|Z|<200 \mathrm{pc}$ and arrived at $Z_{0}=27 \pm 5 \mathrm{pc}$. His study of the distribution of distant $\mathrm{OB}$ stars yielded $Z_{0}=2 \pm 9 \mathrm{pc}$. Stothers and Frogel (1974) deduced the mean plane from O-B5 stars, B0-B5 Be stars, O-M supergiants, clusters and associations. Their values for the sun's elevation above the plane were $24 \pm 3,46 \pm 15,-54 \pm 8$, and $29 \pm 13 \mathrm{pc}$, respectively.

The relative positions of the sun and the mean galactic plane can be deduced from Pioneer 10 brightness data with excellent accuracy. The observation of the average latitude above and below which equal numbers of significant brightness variations occurs $\left(b=-1.4^{\circ} \pm\right.$ 0.3) can be coupled with the theoretical result (Toller, 1981) that the generation of brightness maxima and minima on a spatial scale of several degrees generally occurs at distances of $450 \pm 125 \mathrm{pc}$. This implies that our line of sight crosses the galactic plane at a distance of 450 pc in the direction $b=-1.4^{\circ}$, resulting in $Z_{0}=11 \pm 3.9 \mathrm{pc}$. Similarly, the background light is generally brighter at latitude $-b$ than $+b$. The brightness symmetry plane at $b=-2.0 \pm 0.3$ leads to $Z_{0}=15.7 \pm 4.9 \mathrm{pc}$. Finally, the Pioneer data indicates an absence of peaks and dips at $b>30^{\circ}, b<-38^{\circ}$. This implies that the amount of material along these two cones of sight are comparable. Assuming a dust distribution proportional to $e^{-Z / \beta}$, with the dust scale height $\beta$ equal to $120 \mathrm{pc}$, produces $Z_{0}=13.1 \pm 10.8$. A weighted average of these three methods results in $Z_{0}=12.8 \pm 2.9 \mathrm{pc}$.

\section{GALAXY MODELS}

Galaxy models are powerful tools for ascertaining Milky Way characteristics. The brightness levels measured by Pioneer have been reproduced to 5\% accuracy by Toller (1981) using exponential scale heights for 33 spectral-luminosity classes of stars and a radiative transfer code. This represents a closer fit than the Caplan and Grec (1979) or Mattila (1980) models. A more detailed study of galactic photometric parameters based, in part, on Pioneer data has been provided by van der Kruit (1986 and this volume). A description of the Milky Way based on photographic and UV photometry has been given by Seidensticker, Schmidt-Kaler, 
and Schlosser (1982), Pröll, Schmidt-Kaler, and Schlosser (1983), Schmidt-Kaler et al. (1983), and Winkler, Pfleiderer, and Schmidt-Kaler (1984).

Galaxy models based on star counts have been formulated by de Vaucouleurs and Pence (1978), van den Bergh (1980), Bahcall and colleagues (1980, 1983a, 1983b, 1984), Gilmore (1981), Gilmore and Reid (1983), Pritchet (1983), Edmunds and Phillipps (1984), Buser and Kaeser (1984), and Sandage (1987). Typical model parameters include the sun's galactocentric distance, a disk scale length, a disk scale height, the disk luminosity function, the colormagnitude diagram, and a spheroid axial ratio. Disk and spheroid components are required to meet observational constraints. A central bulge and low-luminosity halo are added to explain the rotation curve. Bahcall and co-workers assume the general shape of the galaxy by analogy to other galaxies, but use star counts to fix other parameters. The derived photometric parameters are described in the above references and by Scheffler (1982).

\section{FUTURE DIRECTIONS}

Some of the concerns with using currently available star count data to derive knowledge of galactic structure, integrated starlight, and diffuse galactic light are the sparseness of well calibrated data, discrepancies between various studies, and the non-uniformity of extinction and stellar distribution. Star counts using Schmidt-type telescopes have been reviewed by Kron (1984). The development and use of high-speed automated measuring devices, capable of performing star magnitude counts over large areas, should overcome past obstacles. These devices are used extensively at the Space Telescope Science Institute, the University of Minnesota, The Royal Observatory Edinburgh, Cambridge, and elsewhere. They are described by Stobie and McInnes (1982) and Klinglesmith (1983). Their application to star counts has begun (Tanabe and Mori, this volume; Stobie and Ishida, 1987; Jarvis and Tyson, 1981; Irwin and Trimble, 1984). Parameters useful for distinguishing between star and galaxy images are discussed by Kurtz (1983). Deep star counts in small areas (Reid and Gilmore, 1982; Irwin and Trimble, 1984; Gilmore, Reid, and Hewett, 1985) supplement the observational material by which galaxy models are constrained. The launch of the Hubble Space Telescope will open another frontier for galactic studies.

Acknowledgments - I would like to thank Kalevi Mattila and Christoph Leinert for enabling my participation in this conference.

\section{REFERENCES}

Bahcall, J. N., and Soneira, R. M. 1980, Ap. J. Suppl., 44, 73.

Bahcall, J. N., Schmidt, M., and Soneira, R. M. 1983, Ap. J., 265, 730.

Bahcall, J. N., and Soneira, R. M. 1983, in Kinematics, Dynamics and Structure of the Milky Way, ed. W. L. H. Shuter (Dordrecht: Reidel), 209.

Bahcall, J. H., and Soneira, R. M. 1984, Ap. J. Suppl., 55, 67.

Behr, A. 1965, Zeit. Astrophys., 62, 157.

Blaauw, A. $1960, M . N$. R. A. S., 121, 164.

Blaauw, A., and Schmidt, M. 1965, ed. Galactic Structure (Chicago: University of Chicago Press).

Bok, B. J. 1931, Harvard Collage Obs. Circ., 371.

Bok, B. J. 1937, The Distribution of the Stars in Space (Chicago: University of Chicago Press).

Bottlinger, K. F. 1932, Zeit. Astrophys., 4, 370.

Boughn, S. P., and Kuhn, J., R. 1986, Ap. J., 309, 33.

Buser, R., and Kaeser, U. 1984, Astr. Ap., 145, 1.

Caplan, J., and Grec, G. 1979, Astr. Ap., 14, 335.

Classen, C. 1976. Ph.D. Thesis, Bonn.

Davies, R. D. 1960, M. N.R. A. S., 120, 483. 
De Vaucouleurs, G. 1949, Ann. Astrophys., 12, 162.

De Vaucouleurs, G., and Pence, W. D. 1978, A. J., 83, 1163.

Dube, R. R., Wickes, W. C., and Wilkinson, D. T. 1977, Ap. J., 215, L51.

Dumont, R., Soulie, G. Rapaport, M., and Sanchez-Martinez, F. 1970, Astrophys. Space Sci., 9, 175.

Edmunds, M. G., and Phillipps, S. 1984, Astr. Ap., 131, 169.

Elsässer, H., and Haug, U. 1960, Zeit. Astrophys., 50, 121.

Elsässer, H., Neckel, Th., and Scheffler, H. 1965, Zeit. Astrophys., 63, 1.

Elvey, C. T., and Roach, F. E. 1937, Ap. J., 85, 213.

Eroshevich, E. S. 1978, Akademiia Nauk Kazakhskoi SSR, Alma-Ata Astrofiz. Inst. Trudy, 31, 26.

FitzGerald, M. P. 1968, A. J., 73, 983.

Gilmore, G. 1981, M. N. R. A. S., 195, 183.

Gilmore, G., and Reid, N. 1983, M. N. R. A. S., 202, 1025.

Gilmore, G., Reid, N., and Hewett, P. 1985, M. N. R. A. S., 213, 257.

Gum, C. S., Kerr, F. J., and Westerhout, G. 1960, M. N. R. A. S., 121, 132.

Hanner, M. S., Leinert, C., and Pitz, E. 1978, Astr. Ap., 65, 245.

Hanner, M. S., Weinberg, J. L., DeShields II, L. M., Green, B. A., and Toller, G. N. 1974, J. Geophys. Res., 79, 3671.

Heiles, C. 1975, Astr. Ap. Suppl., $20,37$.

Henyey, L. G., and Greenstein, J. L. 1940, Ann. Astrophys., 3, 117.

Henyey, L. G., and Greenstein, J. L. 1941, Ap. J., 93, 70.

Irwin, M. J., and Trimble, V. 1984, A. J., 89, 83.

Isserstedt, J., and Schmidt-Kaler, Th. 1964, Zeit. Astrophys., 59, 182.

Jarvis, J. F., and Tyson, J. A. 1981, A. J., 86, 476.

Kirschner, R. P., Oemler Jr., A., and Schechter, P. L. 1979, A. J., 84, 951.

Klinglesmith, D. A. ed. 1983, NASA Conf. Publ. 2317, Astronomical Microdensitometry Conference (Greenbelt: NASA).

Kondraijeva, D. N. 1971, Akademiia Nauk Kazakhskoi SSR, Alma-Ata, Trudy Astrofiz., 16, 22.

Krautter, J. 1980, Astr. Ap., 89, 74.

Kreiken, E. A. 1936, Zeit. Astrophys., 12, 340.

Kreiken, E. A. 1941, Ap. J., 94, 259.

Kron, R. G. 1984, in IAU Colloquium 78, Astronomy with Schmidt-Type Telescopes, ed. M. Capaccioli (Dordrecht: Reidel), 315.

Kurtz, M. J. 1983, in Statistical Methods in Astronomy, ESA Special Publication 201, 47.

Lillie, C. F. 1968, Ph.D. Thesis, University of Wisconsin.

Lillie, C. F., and Witt, A. N. 1976, Ap. J., 208, 64.

Mattila, K. 1970, Astr. Ap., 8, 273.

Mattila, K. 1976, Astr. Ap., 47, 77.

Mattila, K. 1980, Astr. Ap., 82, 373.

Mattila, K. 1989, in Proceedings of IAU 139, Galactic and Extragalactic Background Radiation, ed. S. Bowyer and Ch. Leinert, Dordrecht, Kluwer Academic Publisher.

Megill, L. R., and Roach, F. E. 1961, NBS Tech. Note \#106, U.S. Dept. of Commerce, Office of Technical Services.

Neckel, Th. 1967, Landessternhwarte Heidelberg-Königstuhl Verofentlichungen, 19.

Neckel, Th. 1968, Zeit. Astrophys., 69, 112.

Pannekoek, A. 1933, Publ. of the Astron. Inst. of the Univ. of Amsterdam, Number 3.

Pannekoek, A., and Koelbloed, D. 1949, Publ. of the Astron. Inst. of the Univ. of Amsterdam, Number 9.

Pavlovskaya, E. D., and Sharov, A. S. 1971, Sov. Astr., 14, 849.

Peters, G. 1970, Astr. Ap., 4, 134.

Peterson, B., Ellis, R., Kibblewhite, E., Bridgeland, M., Hooley, T., and Horne, D. 1979, Ap. J., 233, L109.

Pritchet, C. 1983, A. J., 88, 1476.

Pröll, H. J., Schmidt-Kaler, Th., and Schlosser, W. 1983, Astr. Ap. Suppl., 51, 17.

Reid, N., and Gilmore, G. 1982, M. N. R. A. S., 201, 73.

Roach, F. E. 1964, Space Sci. Rev., 3, 512.

Roach, F. E. 1967, in Modern Astrophysics, ed. M. Hack (Paris: Gauthiers-Villars), 49. 
Roach, F. E., and Megill, L. R. 1961, Ap. J., 133, 228.

Roach, F. E., and Smith, L. L. 1964, NBS Tech. Note 214.

Roach, F. E., and Smith, L. L. 1968, Geophys. J. R. A. S., 15, 1.

Roach, F. E., Smith, L. L., Pfleiderer, J., Batishko, C., and Batishko, K. 1972, Ap. J., 173, 343.

Sandage, A. 1987, A. J., 93, 610.

Scheffler, H. 1982, in Landolt-Bornstein Vol. 2C, ed. K.-H. Hellwege, K. Schaifers, and H. H. Voigt (Berlin: Springer-Verlag), 193.

Schmidt, T., and Leinert, C. 1966, Zeit. Astrophys., 64, 110.

Schmidt-Kaler, Th., Seidensticker, K. H., Pröll, H. J., Schlosser, W., and Beck, R. 1983, Astr. Ap. Suppl., 51, 1.

Seares, F. H., van Rhijn, P. H., Joyner, M. C., and Richmond, M. L. 1925, Ap. J., 62, 320.

Seidensticker, K. J., Schmidt-Kaler, T., and Schlosser, W. 1982, Astr. Ap., 114, 60.

Shane, C. D., and Wirtanen, C. A. 1967, Publ. of the Lick Obs., 22, part 1.

Sharov, A. S. 1964, Sov. Astr., 7, 689.

Sharov, A. S. 1971, Sov. Astr., 14, 942.

Sharov, A. S., and Lipaeva, N. A. 1973, Sov. Astr., 17, 69.

Sparrow, J. G., and Ney, E. P. 1972, Ap. J., 174, 717.

Spinrad, H., and Stone, R. 1978, Ap. J., 226, 609.

Sternberg, J. R., and Ingham, M. F. 1972, M. N. R. A. S., 159, 1.

Stobie, R. S., and McInnes, B., 1982, ed. Workshop on Astronomical Measuring Machines, Occasional Reports of the Royal Observatory, Edinburgh.

Stobie, R. S., and Ishida, K. 1987, A. J., 93, 624.

Stothers, R., and Frogel, J. A. 1974, A. J., 79, 456.

Tanabe, H. 1973, World Data Center C2 (Airglow), Tokyo Astr. Obs., Mitaka, Japan, 48.

Tanabe, H., and Mori, K. 1971, Publ. of the Roy. Obs., Edinburgh, 8, 173.

Tanabe, H., and Mori, K. 1976, in IAU Colloquium 31, Interplanetary Dust and Zodiacal Light, Lecture Notes in Physics 48, ed. H. Elsässer and H. Fechtig (Heidelberg: Springer-Verlag), 36.

Tanabe, H., and Mori, K. 1979, personal communication.

Tanabe, H., and Mori, K. 1989, in Proceedings of IAU 139, Galactic and Extragalactic Background Radiation, ed. S. Bowyer and Ch. Leinert, Dordrecht, Kluwer Academic Publisher.

Toller, G. N. 1981, Ph.D. Thesis, State University of New York at Stony Brook.

Toller, G. N. 1983, Ap. J., 266, L79.

Toller, G. N., Tanabe, H., and Weinberg, J. L. 1987, Astr. Ap., 188, 24.

Tyson, J. A. 1988, A. J., 96, 1.

Tyson, J. A. 1989, in Proceedings of IAU 139, Galactic and Extragalactic Background Radiation, ed. S. Bowyer and Ch. Leinert, Dordrecht, Kluwer Academic Publisher.

Tyson, J. A., and Jarvis, F. J. 1979, Ap. J., 230, L153.

Uranova, T. A. 1969, Sov. Phys.-Astr., 12, 1041.

Van den Bergh, S. 1980, in IAU Colloquium 54, Scientific Research with the Space Telescope (NASA CP-2111), ed. M. S. Longair and J. W. Warner (Washington, DC: Government Printing Office), 151.

Van der Kruit, P. C. 1986, Astr. Ap., 157, 230.

Van der Kruit, P. C. 1989, in Proceedings of IAU 139, Galactic and Extragalactic Background Radiation, ed. S. Bowyer and Ch. Leinert, Dordrecht, Kluwer Academic Publisher.

Van Houten, C. J. 1961, Bull. Astr. Inst. Neth., 19, 303.

Van Rhijn, P. J. 1925, Gröningen Publication No. 43, Kapteyn Astron. Lab., Gröningen, Netherlands.

Van Tulder, J. J. M. 1942, Bull. Astr. Inst. Neth., 9, 315.

Weinberg, J. L. 1969, Bull. A. A. S., 1, 368.

Weinberg, J. L. 1981, Sky and Tel., 61, 114.

Weinberg, J. L., Hanner, M. S., Beeson, D. E., DeShields II, L. M., and Green, B. A. 1974, J. Geophys. Res., 79, 3665.

Winkler, C. Pfleiderer, J., and Schmidt-Kaler, Th. 1984, Astr. Ap. Suppl., 58, 705.

Witt, A. N. 1968, Ap. J., 152, 59.

Witt, A. N., and Lillie, C. F. 1973, Astr. Ap., 25, 397.

Wolstencroft, R. D., and Rose, L. J. 1966, Nature, 209, 388.

Zavarzin, Yu. M. 1978, Akademiia Nauk Kazakhskoi SSR, Alma-Ata, Astrofiz. Inst. Trudy, 31, 31. 
Ch. Leinert: Given the 14\% calibration difference between Helios and Pioneer, could you comment on the reliability of the extragalactic component derived by subtraction?

G. Toller: The relative calibration between Helios and Pioneer needs to be investigated further by comparing observations made from near $1 \mathrm{AU}$ along similar lines of sight and performing corrections for dust symmetry plane, radial distance from the sun, and bandpass effects. The higher brightness seen from the Pioneer space probe is necessary to obtain non-negative values for the extragalactic background at high galactic latitudes. The error in the determination of extragalactic background light due to photometric and star count uncertainties is diminished by considering observations at many high galactic latitude regions. The $1 \sigma$ error is $1.3 \mathrm{~S}_{10}(V)$ at $440 \mathrm{~nm}$ for Pioneer-based measurements.

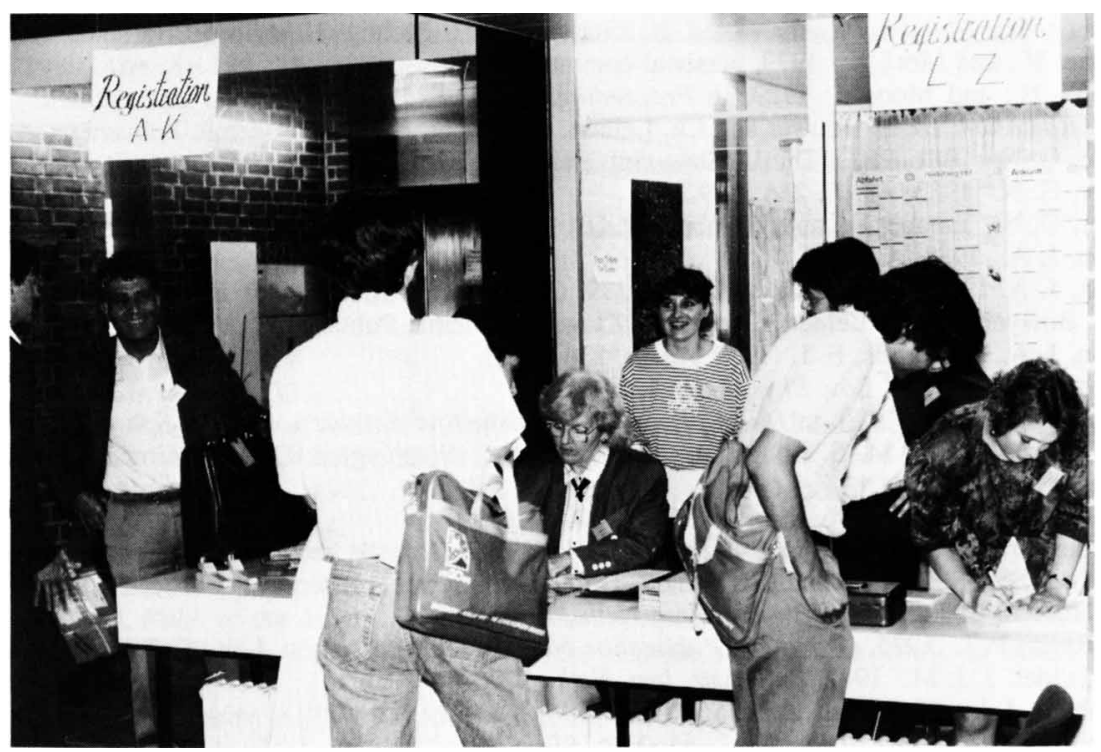

Registration 